\title{
Structural Determinants of Lateral Gate Opening in the Protein Translocon ${ }^{\dagger}$
}

\author{
James Gumbart and Klaus Schulten* \\ Department of Physics, University of Illinois at Urbana-Champaign, Beckman Institute for Advanced Science and Technology, \\ Urbana, Illinois 61801
}

Received May 3, 2007; Revised Manuscript Received June 25, 2007

\begin{abstract}
The heterotrimeric SecY/Sec61 complex is a protein-conducting channel that provides a passage for proteins across the membrane as well as a means to integrate nascent proteins into the membrane. While the first function is common among membrane protein channels and transporters, the latter is unique. Insertion of nascent membrane proteins, one transmembrane segment at a time, by SecY likely occurs through a lateral gate in the channel. Molecular dynamics simulations have been used to investigate the mechanism of gate opening. Opening and closing the gate under different conditions allowed us to identify structural elements that resist opening as well as those that aid closure. SecE, considered to act as a clamp keeping the lateral gate closed, was found to play no such role. Loosening of the plug by lateral gate opening, a potential step in channel gating, was also observed. The simulations revealed that lipids on time scales of up to $1 \mu$ s do not flood channels with an open lateral gate.
\end{abstract}

Protein trafficking by the Sec pathway is a cellular process common to all domains of life. Newly formed (or forming), unfolded proteins are targeted by means of an N-terminal signal sequence to the Sec translocon in the endoplasmic reticulum membrane (in eukaryotes) or the cytoplasmic membrane (in prokaryotes) $(1-6)$. In the membrane, the translocation channel, the SecY or Sec61 complex, either permits the nascent polypeptide to cross the membrane or integrates it into the membrane. This translocon performs its function in concert with a variety of other macromolecular assemblies: the ribosome, $\mathrm{SecA}$ (in bacteria) or $\mathrm{BiP}$ (in eukaryotes), and others. These macromolecules provide the driving force necessary to feed the polypeptide through the channel and prevent it from sliding back.

The protein translocation channel is comprised of three subunits, two of which are well-conserved. These two, known as $\operatorname{Sec} \mathrm{Y}$ and SecE in prokaryotes (Sec61 $\alpha$ and Sec61 $\gamma$ in most eukaryotes), form the basic unit for translocation of polypeptides (see, for example, the reviews by Osborne et al. and Pohlschröder et al. in refs 2 and 3). An additional subunit, $\operatorname{SecG}$ in bacteria and $\operatorname{Sec} \beta$ in archaea $(\operatorname{Sec} 61 \beta$ in eukaryotes), shows no homology between bacteria and archaea/eukarya, and while it promotes translocation, it is not required for it $(2,7,8)$. The crystallographic structure of SecYE $\beta$ from Methanococcus jannaschii revealed in detail the arrangement of the three subunits and hinted at the function of different structural elements (8).

For the translocation of nascent proteins, a channel through the center of a single $\operatorname{Sec} Y$ was proposed $(8,9)$; this hourglass-shaped channel is closed by both a hydrophobic

$\lceil$ This work was supported by the National Institutes of Health (P41RR05969 and R01-GM067887). The authors gratefully acknowledge computer time provided by the Pittsburgh Supercomputer Center and the National Center for Supercomputing Applications through the National Resources Allocation Committee (MCA93S028).

* To whom correspondence should be addressed. Telephone: (217) 244-1604. Fax: (217) 244-6078. E-mail: kschulte@ks.uiuc.edu. ring of residues near the center as well as by a small, helical "plug" domain blocking the periplasmic half-channel. Previous simulations indicated that the pore ring is flexible yet maintains a tight seal sufficient to block the passage of small molecules and ions (10); however, the absence of the plug domain may increase the flexibility of the pore ring and even the channel as a whole (11).

Integration of membrane proteins into the bilayer is proposed to occur one transmembrane domain $(\mathrm{TM})^{1}$ at a time through a lateral gate in SecY, located at the interface of two halves of the structure, between TMs $2 b / 3$ and $7 / 8$ (see Figure 1). Extensive experiments characterizing the probability of helix insertion through SecY into the membrane demonstrated a correlation with the Wimley-White hydrophobicity scale, suggesting that the inserting TM samples the lipid phase directly while still within the channel $(12,13)$, although such results may neglect additional factors governing insertion (14). Other experiments support a sequential insertion into the bilayer but suggest different TMs (and accessory proteins) influence each other during the integration process (15-17). Cross-linking experiments, performed for the insertion of the membrane proteins opsin (17) and aquaporin (16), demonstrated that each TM separately inserts into the bilayer and that some TMs do so more easily than others. Some TMs appear to require the insertion of the next TM in sequence to move into the bilayer, while others do not; also, TMs sometimes leave the vicinity of the translocon during insertion but then return at a later stage, possibly to aid insertion of the subsequent TM (16, 17). Recent experiments have shown that the helices of the voltage sensor of the Shaker $\mathrm{K}^{+}$channel also insert cooperatively but not individually (18). Atomic force microscopy experiments in which helices of bacteriorhodopsin are pulled

\footnotetext{
${ }^{1}$ Abbreviations: MD, molecular dynamics; SMD, steered molecular dynamics; CG, coarse grained; rmsd, root-mean-square deviation; TM, transmembrane domain.
} 


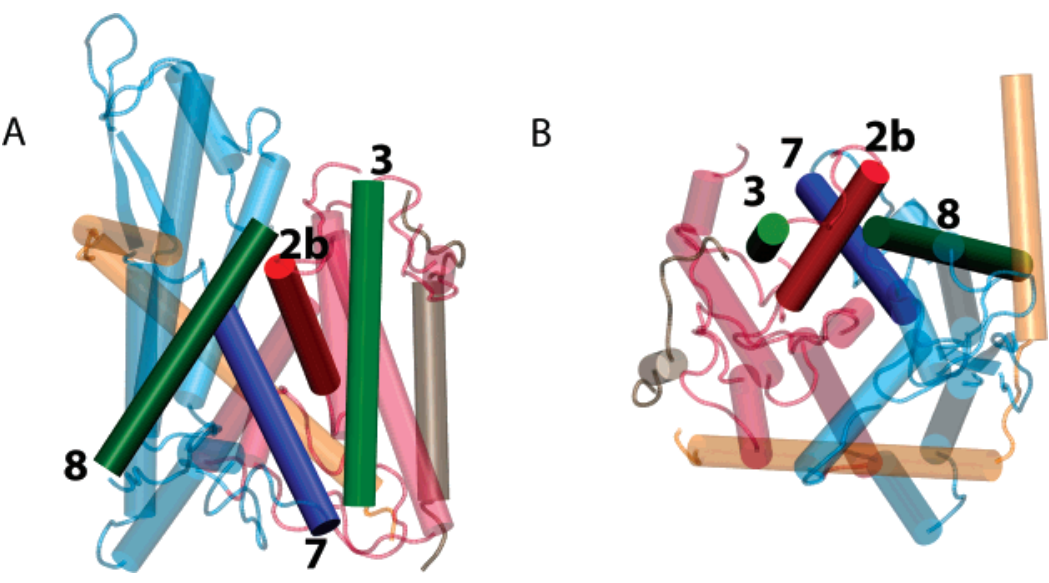

FIGURE 1: $\operatorname{SecYE} \beta$ viewed in a representation showing helices as cylinders. The two pseudosymmetric halves of SecY, TMs $1-5$ and 6-10 (PDB code 1RHZ), are shown in red and blue, respectively. SecE is shown in orange, and Sec $\beta$ is shown in brown. All of the protein is transparent except for the helices that comprise the lateral gate: $2 \mathrm{~b}$ (red), 3 (green), 7 (blue), and 8 (dark green). (A) View from the membrane plane. (B) View from the cytoplasmic side.

out of the membrane, a process reverse to integration, show an interesting resemblance to the integration behavior; when pulled from the membrane, the helices unfold through different pathways, sometimes sequentially or pairwise ( 19 , 20).

The functional role of the translocon monomer is controversial, with experiments suggesting that only oligomers are functional. While dimers $(21-23)$ and tetramers $(24,25)$ of the Sec complex have been observed, different associations have been proposed. One arrangement, a back-to-back dimer based on an $8 \AA$ cryo-EM map of a SecYEG dimer (21) and cross-linking data linking two SecEs $(26,27)$, places the lateral gate of each SecY facing out, away from the interface $(8,28)$. The reason for oligomerization in this arrangement has been hypothesized to be cooperative interactions between monomers, including those beneficial to translocation directly and for recruiting and binding channel partners $(2,8,10$, 28). Another arrangement, a front-to-front dimer based on 3D cryo-EM reconstructions, places the lateral gates facing each other (23). Such an arrangement could provide for a larger, consolidated channel when necessary, by opening both lateral gates simultaneously; this arrangement could also provide two separate pathways, one channel for the soluble sections and one for the membrane sections of a nascent protein, with the decision of which channel to use being made by the ribosome $(29,30)$.

In the present study, we provide a detailed, dynamical examination of the feasibility and mechanism of $\operatorname{SecYE} \beta$ lateral gate opening and closing by performing extensive allatom molecular dynamics (MD) simulations on the channel in a fully solvated lipid bilayer. Because the oligomeric state is still unknown and previous simulations of the monomer suggested it can, in principle, act independently (10), we chose to focus on the $\operatorname{SecYE} \beta$ monomer only. Using steered MD (SMD), the lateral gate of SecY is forced open and then allowed to relax afterward while we examine the structural behavior during these processes, finding that both occur without large structural deformations to the protein. The connections of SecE and the plug domain to lateral gate opening and closing are also investigated by removing them and repeating the simulated opening and relaxation, with the former being particularly important because the effect of removing SecE cannot be tested directly experimentally as a result of the channel being nonfunctional in vivo without it. Finally, the behavior of lipids near the gate is explored through simulations holding the gate open, both in an allatom representation and a coarse-grained (CG) representation, which permits time scales normally out of the range of conventional MD (see the Materials and Methods).

\section{MATERIALS AND METHODS}

Simulated System. Modeling of $\operatorname{Sec} \mathrm{YE} \beta$ began with the $3.5 \AA$ resolution coordinates obtained from the Protein Data Bank (PDB code 1RHZ) (8). The protein was placed in a pre-equilibrated 1-palmitoyl-2-oleoyl-sn-glycero-3-phosphocholine (POPC) lipid bilayer membrane and solvated with water above and below using plugins in VMD (31). The resulting system was complemented through the inclusion of $\mathrm{Na}^{+}$and $\mathrm{Cl}^{-}$ions to a concentration of $50 \mathrm{mM}$, making the net charge zero. In total, the protein/lipid/water system contained 106679 atoms and was approximately $100 \AA$ in length along all dimensions. The system was equilibrated over $5 \mathrm{~ns}$ in a multistage process; further details are described in ref 10 .

All-Atom MD. MD simulations were carried out using NAMD 2.5/2.6 (32) and the CHARMM27 force field (33). A multiple-time-stepping algorithm was used to efficiently evaluate electrostatic interactions. Bonded interactions were computed every $1 \mathrm{fs}$; short-range nonbonded interactions were computed every $2 \mathrm{fs}$; and long-range interactions (defined as beyond a $12 \AA$ cutoff) were computed every 4 fs. The particle-mesh Ewald (PME) method with a grid density of more than $1 / \AA^{3}$ was used to calculate long-range electrostatics without requiring a cutoff.

A constant temperature was enforced for all simulations at $T=310 \mathrm{~K}$ using Langevin dynamics coupled lightly (damping coefficient of $1.0 \mathrm{ps}^{-1}$ ) to the oxygen atoms of water molecules. A constant pressure of $1 \mathrm{~atm}$ was also maintained in all periodic boundary simulations using a Nosé-Hoover Langevin piston; in all cases, the dimensions of the unit cell were allowed to fluctuate independently. When periodic boundary conditions were employed, the distance between protein images in neighboring cells was never less than approximately $25 \AA$.

$C G M D$. CG MD is a technique to reduce the spatial complexity of a system to increase accessible simulation time 


\begin{tabular}{|c|c|c|c|}
\hline simulation name & length (ns) & start & description \\
\hline sim1a & 6 & $\operatorname{Sec} \mathrm{YE} \beta$ & opening the lateral gate (ran twice) \\
\hline $\operatorname{sim} 1 b$ & 18 & $\operatorname{SecYE} \beta$ & opening the lateral gate (slow) \\
\hline $\operatorname{sim} 2 \mathrm{a}$ & 6 & $\operatorname{Sec} \mathrm{Y} \beta$ & opening the lateral gate \\
\hline $\operatorname{sim} 3 \mathrm{a}$ & 6 & $\operatorname{SecYE} \beta \Delta$ plug & opening the lateral gate (ran twice) \\
\hline $\operatorname{sim} 1 \mathrm{c}$ & 15 & $\operatorname{sim} 1 \mathrm{a}$ & relaxation (gate closure) \\
\hline $\operatorname{sim} 2 b$ & 15 & $\operatorname{sim} 2 \mathrm{a}$ & relaxation (gate closure) \\
\hline $\operatorname{sim} 3 b$ & 8.2 & $\operatorname{sim} 3 \mathrm{a}$ & relaxation (gate closure) \\
\hline sim $1 d$ & 7.8 & sim $1 \mathrm{a}$ & relaxation with lipids restrained \\
\hline simle & 20 & $\operatorname{sim} 1 \mathrm{a}$ & holding gate open (all atom) \\
\hline $\operatorname{sim} 1 C G$ & 1000 & sim1a & holding gate open (CG) \\
\hline $\operatorname{sim} 1 g$ & 15 & sim1e & relaxation (gate closure) \\
\hline $\operatorname{sim} 1 \mathrm{~h}$ & 3 & $\operatorname{sim} 1 \mathrm{a}$ & holding the gate at an intermediate opening \\
\hline $\operatorname{sim} 4$ & 6 & & separation of gate helices extracted from $\operatorname{Sec} \mathrm{YE} \beta$ \\
\hline $\operatorname{sim} 5$ & 2.4 & & equilibration of a bilayer with a hole \\
\hline $\operatorname{sim} 1 \mathrm{a}-20$ & 6 & $\operatorname{SecYE} \beta$ & repeat of sim 1 a (see the Supporting Information) \\
\hline $\operatorname{sim} 1 \mathrm{a}-50$ & 6 & $\operatorname{SecYE} \beta$ & repeat of sim1a (see the Supporting Information) \\
\hline $\operatorname{sim} 1 \mathrm{a}-515$ & 6 & $\operatorname{SecYE} \beta$ & repeat of sim1a with a larger bilayer (see the Supporting Information) \\
\hline
\end{tabular}

${ }^{a}$ Simulations are listed with their name and conditions. $\operatorname{sim} 1$ denotes simulations of the native $\operatorname{SecYE} \beta$; $\operatorname{sim} 2$ refers to the system without SecE $(\operatorname{Sec} Y \beta)$; and $\operatorname{sim} 3$ refers to the system without the plug $(\operatorname{SecYE} \beta \Delta$ plug). The state of the protein used to begin each simulation is listed under the start column; in cases where simulations began from the equilibrated structure, the protein itself is listed.

scales. The atoms in biological molecules are mapped to a reduced (relative to the number of atoms) set of beads in the following way: amino acid residues are each represented by two beads (except glycine), with one bead representing the backbone and one representing the side chain; POPC lipids are represented through 13 beads; and four water molecules are represented through one bead. The mass of each bead is the sum of its constituent atoms. Parameters for lipids and water were developed by Marrink et al. (34), and parameters for proteins were developed by Shih et al. (35). Protein parameters were initially developed on the basis of all-atom structures and simulations (35), and some were later refined further (36). The structure of the potential function is similar to the one of the all-atom CHARMM potential (35). After coarse-graining, the simulated system contained 9020 beads, over a factor of 10 less than the original 106679 atoms.

NAMD 2.5 was also used to perform the CG simulation, sim1CG (see Table 1). Because the beads move more slowly than atoms as a result of their larger masses and because the potential energy functions are smoother, a longer integration time step (15 fs) can be used for integrating the equations of motion. The smoother potentials also increase the speed of relaxation processes; for example, the selfdiffusion of water is 2-6 times faster in CG than in allatom simulations (34-36). Hence, while $1 \mu$ s of simulation time is reported here, it is representative of a longer time.

A strict cutoff of $12 \AA$ was used with no long-range electrostatic interactions included. To mimic charge screening and polarization effects, all charges are scaled by 0.7 and a relative permittivity of $\epsilon=20$ is used (34). Langevin damping with a constant of $0.5 \mathrm{ps}^{-1}$ was used to keep the system near $323 \mathrm{~K}$, and a constant pressure of $1 \mathrm{~atm}$ was maintained. The entire protein backbone was restrained with a harmonic potential $(k=0.5 \mathrm{kcal} / \mathrm{mol})$ to hold the gate open and avoid any deformations that may obstruct the gate.

SMD Simulations. SMD (37-39) was used to open the lateral gate of $\mathrm{Sec} Y$ at a constant velocity in simulations $\operatorname{sim} 1 \mathrm{a}, \operatorname{sim} 1 \mathrm{~b}, \operatorname{sim} 2 \mathrm{a}, \operatorname{sim} 3 \mathrm{a}$, and $\operatorname{sim} 4$; the simulations are specified in Table 1. Forces in opposite directions were applied individually to the $\mathrm{C}_{\alpha}$ atoms of residues $75,76,79$,
80, 83, 84, 87, 91, and 92 in transmembrane helix TM2b and residues 257, 258, 260, 261, 264, 265, 267, 268, 271, $272,275,276$, and 278 in transmembrane helix TM7, with each set partially lining one side of the gate (see Figure 1). A force constant of $k=2.5 \mathrm{kcal} \mathrm{mol}^{-1} \AA^{-2}$ was employed along with a velocity of $1.5 \AA / n s(0.5 \AA / n s$ in the case of one slower simulation), giving a net opening rate of $3 \AA / n s$ ( $1 \AA / n s$ in the slower simulation). Head groups of lipids on the exterior (46 lipids out of 251 total) were restrained harmonically with a force constant of $k=3.6 \mathrm{kcal} \mathrm{mol}^{-1}$ $\AA^{-2}$. While 251 lipids is a typical amount for a membrane protein simulation, we repeated the gate opening calculation for a system with 515 lipids (simulation sim1a-515) and found the behavior to be similar to that in simla (see the Supporting Information).

For simulations without SecE ( $\operatorname{sim} 2 \mathrm{a}$ and $\operatorname{sim} 2 \mathrm{~b}$; see Table 1), a $\operatorname{SecY} \beta$ system was built starting from the crystal structure and equilibrated for $5 \mathrm{~ns}$ in a procedure similar to that in ref 10 . For simulations involving the removal of the plug, i.e., SecYE $\beta \Delta$ plug ( $\operatorname{sim} 3 a$ and $\operatorname{sim} 3 b$ ), the equilibrated native structure was used as a starting point. Residues $\mathrm{Ala}^{54}$ to $\mathrm{Ile}^{67}$ were removed, and $\mathrm{Pro}^{53}$ was connected to Gly ${ }^{68}$. The resulting structure was minimized for 1000 steps before beginning SMD.

Relaxation. Different approaches were taken during the simulated relaxation of the systems resulting from SMD simulations. For each of the three systems to which SMD was applied (native $\operatorname{SecYE} \beta, \operatorname{SecY} \beta$, and $\operatorname{SecYE} \beta \Delta$ plug), all external forces were turned off and simulations were continued (sim $1 \mathrm{c}, \operatorname{sim} 2 \mathrm{~b}$, and $\operatorname{sim} 3 \mathrm{~b}$; see Table 1). In the case of the native $\operatorname{SecYE} \beta$, two additional simulations were performed. In the first one, sim1e, the gate was fixed in an open position by applying harmonic restraints $(k=5 \mathrm{kcal}$ $\mathrm{mol}^{-1} \AA^{-2}$ ) to the atoms noted above in the description of SMD. In the second case, sim1d, lipids were held fixed by restraining only the phosphorus atom of the headgroup $(k=$ $5 \mathrm{kcal} \mathrm{mol}^{-1} \AA^{-2}$ ). Out of the total 251 lipids, 242 were restrained in this way, with 9 lipids being left free to prevent possible obstruction of the lateral gate, which could affect closing. 


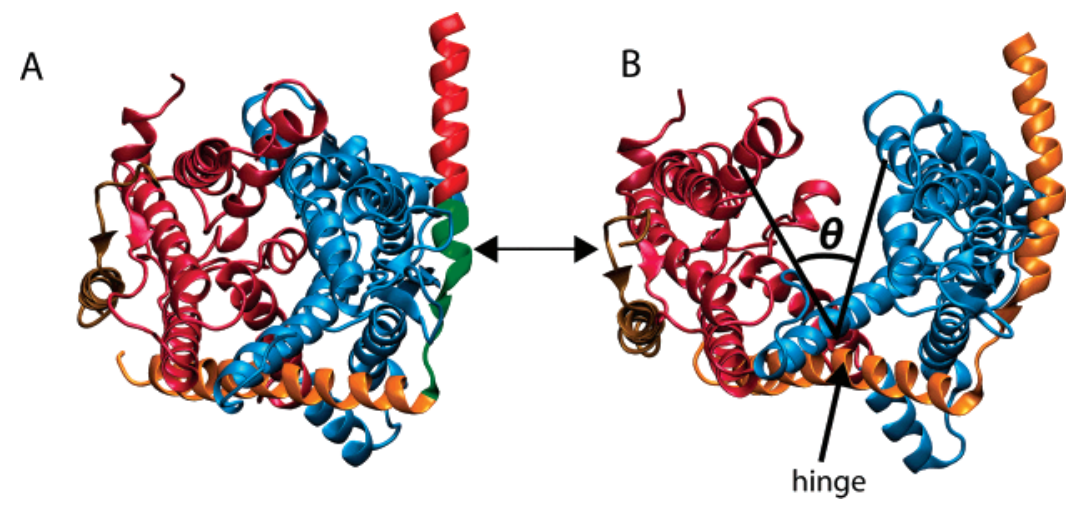

FIgURE 2: Opening the lateral gate of $\operatorname{SecYE} \beta$ viewed from the cytoplasmic side in a cartoon representation. In both A and B, the two pseudosymmetric halves of SecY are shown in red and blue, respectively, and $\operatorname{Sec} \beta$ is shown in brown. (A) Closed state of the lateral gate. $\mathrm{SecE}$ is shown in three colors, with green representing the conserved region of $\operatorname{Sec} \beta$, red representing its nonconserved region, and orange representing its TM helix (see the text). (B) Forced-open state of the lateral gate. The hinge and angle measured are indicated.

Analysis. The total time for the all-atom simulations ( $\sim 100000$ atoms) was approximately $150 \mathrm{~ns}$, and the total time for the $\mathrm{CG}$ simulation ( $\sim 9000$ beads) was $1 \mu \mathrm{s}$. The simulations are listed in Table 1. Simulation trajectory analysis and figure preparation were done using VMD (31). Sequence alignment and comparison was performed with the MultiSeq plugin of VMD (40).

\section{RESULTS}

$\operatorname{SecYE} \beta$, in view of its function, is definitely a highly flexible channel. Naturally, one wishes to explore the mechanisms underlying its largely mechanical function: threading proteins straight through its pore or integrating them sideways into the lipid membrane. This can be done by mechanical manipulation computationally. The computational methodology employed, SMD $(38,41-43)$, has been very successful in confirming and explaining observed mechanical properties of proteins (44), in guiding experimental studies (45), and in predicting properties subsequently observed (46-48). A most recent evaluation of the method is found in Sotomayor and Schulten (49). SMD is the computational equivalent of single-molecule force spectroscopy but provides atomic level pictures of protein mechanics that furnish invaluable guidance to the study of proteins, such as $\operatorname{Sec} \mathrm{YE} \beta$.

Enforced Opening of the Lateral Gate. To investigate the structural characteristics of $\operatorname{Sec} \mathrm{YE} \beta$ when assisting membrane protein insertion, we forced open the lateral gate using SMD (see the Materials and Methods). As illustrated in Figure 2, forces were applied to the channel to mimic the strain that the channel experiences during insertion of a membrane-bound polypeptide. However, it is unknown precisely how the exit process occurs in vivo, which may involve secondary effects arising from channel partners. Regardless, the lateral gate must open to allow for the insertion of TMs, and thus, we believe the process simulated here captures at least the dominant structural results of this opening process on the $\operatorname{SecYE} \beta$ monomer. The simulations carried out are listed in Table 1. One simulation, sim $1 \mathrm{a}$, investigated the complete $\operatorname{Sec} \mathrm{YE} \beta$ complex. Simulation $\operatorname{sim} 2$ a studied $\operatorname{SecY} \beta$ (SecE removed), and $\operatorname{sim} 3 \mathrm{a}$ inspected $\operatorname{SecYE} \beta$ with the plug removed (SecYE $\beta \Delta$ plug).

Given the approximate symmetry between the two halves of SecY (TMs 1-5 and 6-10), we chose to characterize their individual stability. The two halves remain indeed structurally unchanging, despite the forces being placed on each of them; each half maintained its internal secondary and tertiary structure well, in support of the suggestion of a clamshell-like motion of the protein during lateral gate opening (8). As shown in Figure 3, the measured root-meansquare deviation (rmsd) of the protein as a whole grew significantly, in all simulations, as expected because of the opening of the gate changing the conformation of the protein. However, the rmsd of each half of SecY grew minimally in all cases, deviating by only $1-2 \AA$ /atom from the initial geometry. The clamshell-like mechanism of lateral gating is also supported by other simulation studies (50), including one using principle component analysis on an extended equilibrium simulation of $\mathrm{Sec} \mathrm{Y}$ alone (51).

The clamshell-like opening motion of the protein implies the existence of a hinge at the side opposite the gate. The hinge was initially suggested to be located at the loop connecting the two halves, between TMs 5 and 6 (8). Previous simulations also indicated an outward (toward the bilayer) motion of the periplasmic portion of TM 5 when the lateral gate was disturbed (50). The same behavior was observed in our simulations of gate opening with motion of the helix of $2-3 \AA$ in the plane of the membrane. We also located more precisely the hinge region of the protein based on analysis of all simulations by calculating the rmsd per residue over the trajectory and identifying which residues were disturbed the least during gate opening. The linker region between the two halves of TM 5 was found to remain structurally least perturbed, as was the linker between the two helices of SecE and large portions of TM 1 and TM 6 of SecY. Hinge-finding algorithms, Hingefind (52) and Dyndom (53), also place the hinge at the linker region within TM 5. Interestingly, this region (residues Gly ${ }^{188}$, Pro ${ }^{189}$, $\mathrm{Glu}^{190}$, and Gly ${ }^{191}$ ) is not strictly conserved; however, many species exhibit a number of glycines here, indicating that flexibility in the periplasmic end of TM 5 may be functionally relevant.

To determine the major barriers to lateral gate opening, we measured the force required to open the gate. We also identified the structural elements of $\operatorname{SecYE} \beta$ involved in the gating, in particular, SecE and the plug. For this purpose, we carried out constant velocity SMD simulations (see the Materials and Methods). The forces applied, specified in the Materials and Methods, pull $\mathrm{C}_{\alpha}$ atoms on both sides of the gate apart through a spring with moving ends. As the spring 
A 6
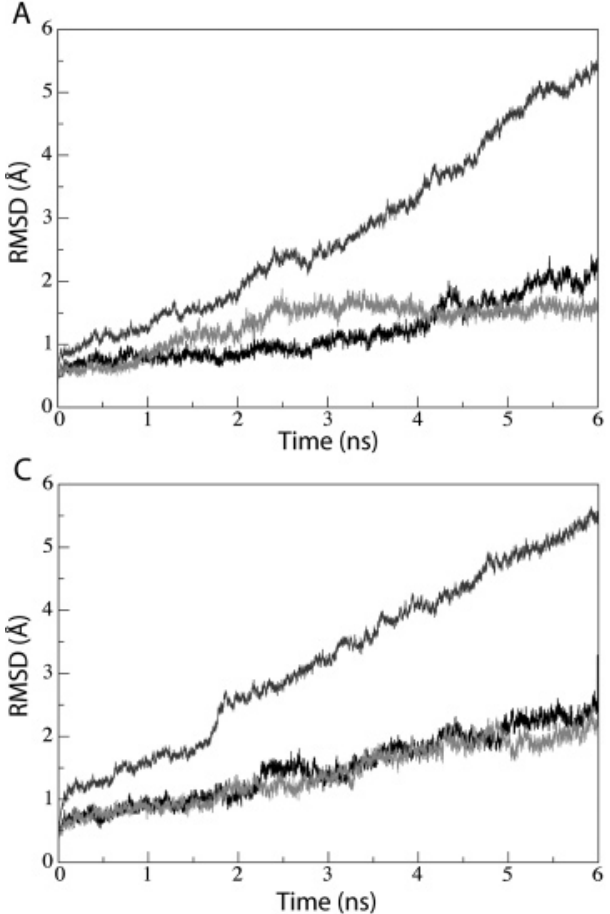

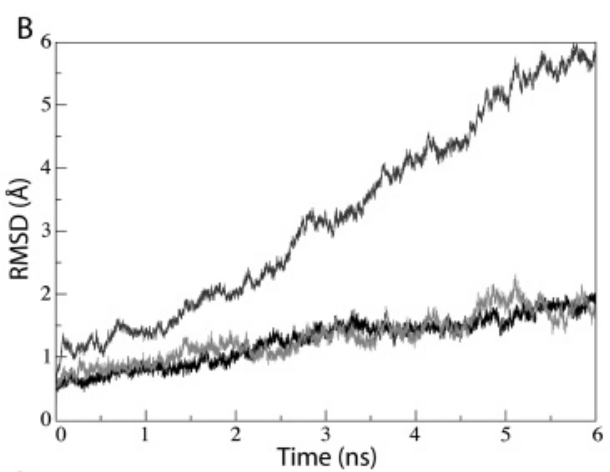

$\mathrm{D}$

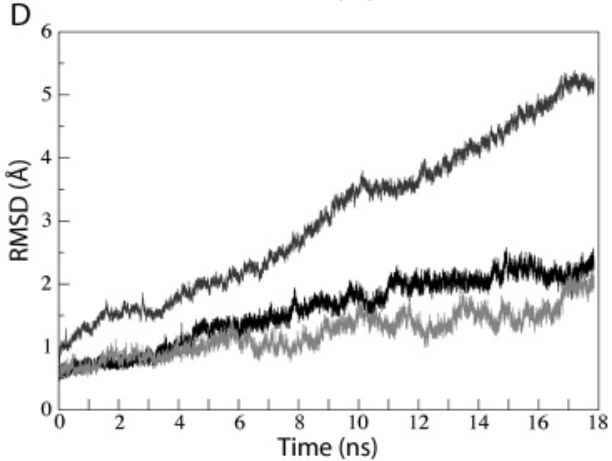

FIGURE 3: Time dependence of the rmsd of the protein during opening. In medium gray is the rmsd for the entire SecY protein backbone as compared to the closed state; in light gray and black are the rmsd values for the two SecY halves (TMs 1-5 and 6-10, respectively, not including connecting loops) as compared to their initial states. Shown are the rmsd values for simulations (A) sim1a (SecYE $\beta$ ), (B) $\operatorname{sim} 2 \mathrm{a}(\operatorname{SecY} \beta),(\mathrm{C}) \operatorname{sim} 3 \mathrm{a}(\operatorname{SecYE} \beta \Delta$ plug), and (D) $\operatorname{sim} 1 \mathrm{~b}$ (SecYE $\beta$ opened at one-third the speed of the others).

extends, forces increase until they are large enough for the gate to open. When the gate experiences increased barriers to opening, forces rise; when barriers are lowered, forces decrease. The gate opening was monitored through the distance $d_{\text {gate }}$ defined as that separating the centers of the two gate helices, TMs $2 b$ and 7. Shown in Figure $4 \mathrm{C}$ is the total applied force $F_{\text {tot }}\left(d_{\text {gate }}\right)$ experienced during gate opening.

The first noticeable peak in the force versus position plot $\left[F_{\text {tot }}\left(d_{\text {gate }}\right)\right]$, shown in Figure 4 , is present for simulations with SecE (sim $1 \mathrm{a}$ and $\operatorname{sim} 3 a)$ but not for one without $(\operatorname{sim} 2 a)$. Also, the overall force profile for simulation sim2a is approximately $20-25 \%$ lower than for the other two systems simulated. The higher forces experienced in sim $1 \mathrm{a}$ and sim $3 \mathrm{a}$ are most likely due to the presence of SecE creating a larger lateral area for the channel as a whole, which must then force a larger fraction of the membrane to accommodate its opening, increasing the force needed for gate opening. Similarly, the force peaks represent a point at which the $\mathrm{N}$-terminal SecE helix begins to bend in response to the membrane pressure.

The presence (or absence) of the plug has a pronounced effect on the opening of $\operatorname{SecYE} \beta$. The force profiles for opening $\operatorname{SecYE} \beta$ (sim1a) and for opening $\operatorname{SecYE} \beta \Delta$ plug (sim3a) are very similar in magnitude (with the latter exhibiting $\sim 10 \%$ lower peak values), with the exception of the region of opening between 6 and $10 \AA$ A Suggested by the plot and confirmed by examination of simulation trajectories, this region corresponds to the separation of the plug from the surrounding portions of SecY. The separation occurs by breaking the hydrophobic contact between the plug and the lateral gate helices, TMs $2 \mathrm{~b}$ and 7 . An additional simulation, sim $1 \mathrm{~h}$, beginning at the point at which the plug became separated ( $3 \mathrm{~ns}$ into sim1a) and holding the gate at its intermediate opening, confirmed that the plug was indeed sufficiently destabilized (the rmsd of the plug is presented in the Supporting Information). The loosening of the plug by moderate lateral gate opening supports the hypothesis that signal sequence intercalation in the lateral gate serves to open the channel by destabilizing interactions between the plug and the rest of $\operatorname{SecY}(2)$.

As seen in Figure 4C, the total force $F_{\text {tot }}$ was measured to be $\sim 2-3 \mathrm{nN}$. When the speed of gate opening was reduced 3 -fold in simulation simlb (from 3 to $1 \AA / \mathrm{ns}$ ), a $15-25 \%$ reduction in force was observed amidst an otherwise similar qualitative behavior. While force peaks late in the simulations ( $d_{\text {gate }}$ between 10 and $15 \AA$ ) can be attributed to protein structural deformation, the majority of the force required to open the gate comes from the displacement of lipids. This was demonstrated through simulation sim4, in which all of the protein $\operatorname{SecYE} \beta$ except the four helices in the gate region ( $2 \mathrm{~b}, 3,7$, and 8 ; see Figure 1) was removed; the remaining four helices were then separated, requiring approximately $1.5-2 \mathrm{nN}$, i.e., the same force as for the full protein, to pull them laterally through the membrane, even after there were no contacts between them. When surface tensions of 20 and $50 \mathrm{dyn} / \mathrm{cm}$ were applied to the system, the force profile was not significantly affected (see the Supporting Information).

Hydrophobic interactions make up the bulk of the contact between the two sides of the lateral gate. Calculations of exposed hydrophobic surface area before and after gate opening indicate a change in the area of approximately 750 $\AA^{2}$; this also accounts for the mitigating effects of lipids, which make contact with the hydrophobic sides of the lateral gate upon opening. Assuming that $15 \mathrm{cal} \mathrm{mol}^{-1} \AA^{-2}$ of free energy is required to expose the hydrophobic surface (54), the change in exposed area would require $\sim 11 \mathrm{kcal} / \mathrm{mol}$ of free energy. This is in notable contrast to a similar calculation, which suggested $65-80 \mathrm{kcal} / \mathrm{mol}$ to be required (29). 

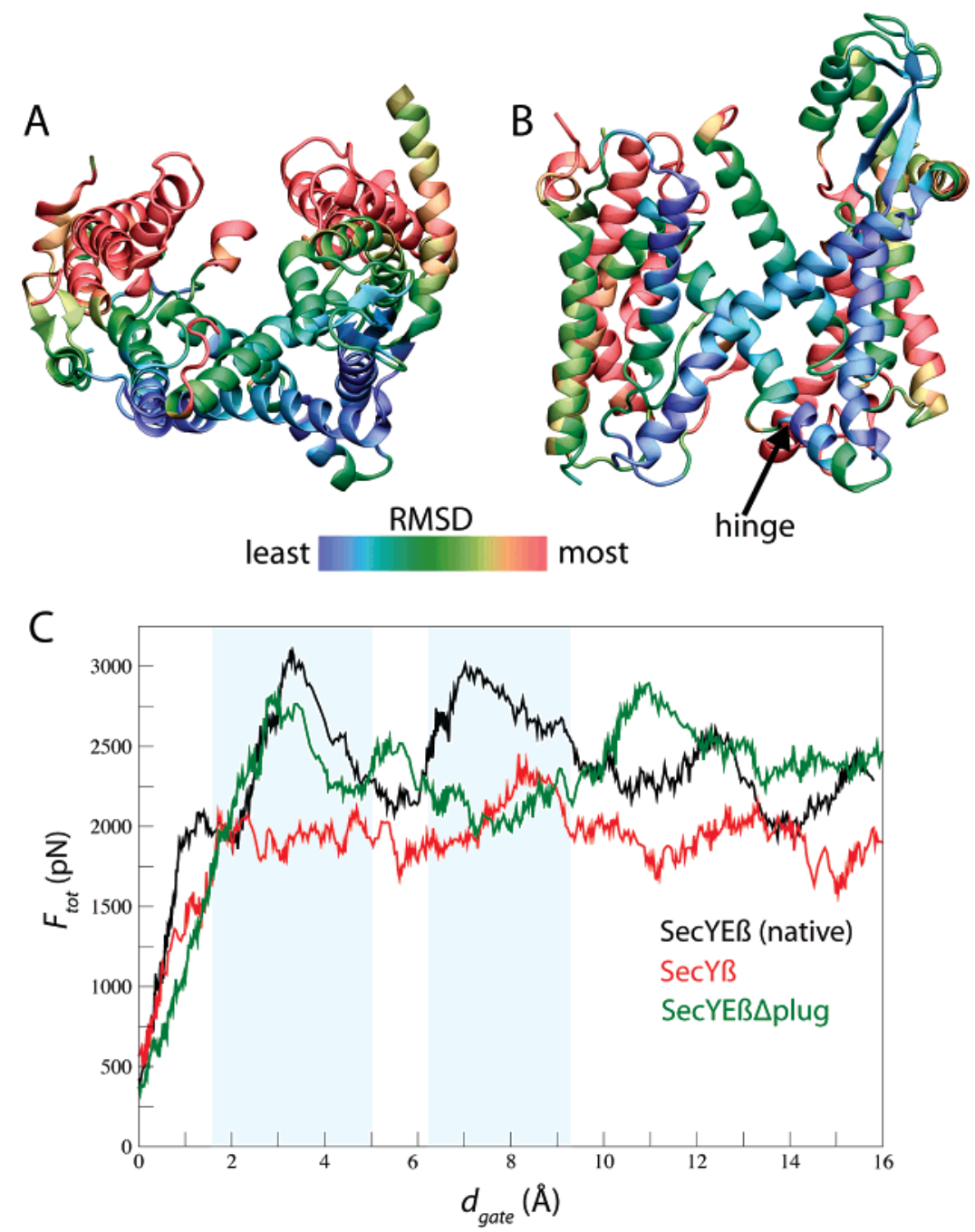

FIGURE 4: Force needed for lateral gate opening and its effect on the structure. In A and $\mathrm{B}, \operatorname{SecYE} \beta$ is shown at the conclusion of lateral gate opening from the (A) cytoplasmic side and (B) within the membrane plane. The protein is colored according to the "stress" at each point, calculated as the rmsd contributed per residue for the entire protein as compared to its initial state. The color scale is also shown with blue indicating very little $(0.4 \AA)$, green indicating moderate, and red indicating large $(14.4 \AA)$ deviation. In B, the determined location of the hinge is indicated. (C) Force required to open the gate as a function of gate opening. The plot shows the force for SecYE $\beta$ (sim1a), $\operatorname{SecY} \beta$ (sim2a), and $\operatorname{SecYE} \beta \Delta$ plug ( $\operatorname{sim} 3 \mathrm{a})$, colored as indicated. The first light blue box denotes the range where the presence of SecE is most noticeable, and the second light blue box denotes the range where the presence of the plug is most pronounced.

The free energy that would be required is further reduced when the smaller opening necessary to free the plug is considered; in that case, the change in the exposed hydrophobic surface area is $283 \AA^{2}$, corresponding to only 4.25 $\mathrm{kcal} / \mathrm{mol}$ of free energy. In addition to hydrophobic interactions, there are three groups of residues that form hydrogen bonds across the gate: $\mathrm{Thr}^{337}$ with $\mathrm{Gln}^{86}$ and Gly ${ }^{90}$ near the cytoplasmic side, $\mathrm{Asn}^{268}$ with $\mathrm{Thr}^{80}$ and $\mathrm{Glu}^{122}$ in the middle, and $\mathrm{Arg}^{278}$ with Gly ${ }^{128}$ near the periplasmic side, with the former two having been recognized previously (8). By measuring the interactions between these three sets of residues over the course of the simulation, we estimate that the buried hydrogen bonds contribute as much as $30 \mathrm{kcal} /$ mol of binding energy; however, this energetic penalty of gate opening is greatly reduced during opening by the formation of new hydrogen bonds with both lipid head groups and water molecules that enter the channel.

Spontaneous Closing of the Lateral Gate. After opening the lateral gate of $\mathrm{SecY}$, each resulting system was allowed to relax (simulated without external forces) for periods lasting between 8 and 15 ns. Simulations sim $1 \mathrm{c}, \operatorname{sim} 2 \mathrm{~b}$, and $\operatorname{sim} 3 \mathrm{~b}$ (see Table 1) examined the stability of the protein during closing as well as the factors that govern closing. Special attention was paid to the role of lipids in closing the gate and to their behavior at the membrane/gate interface.

To quantify the degree of lateral gate closure, the angle formed by the two sides of the gate and the gating hinge was monitored as shown in Figure 5. This angle measured $14^{\circ}$ in the crystal structure and fluctuated only slightly around $15^{\circ}$ during equilibration of the native system (not shown). At the conclusion of all gate-opening simulations, the angle measured $35^{\circ}$. At this angle, the gate was between 10 and $16 \AA$ wide, depending upon the side-chain orientation, which would clearly permit the passage of a transmembrane helix of a typical protein.

Relaxation of the native $\operatorname{SecYE} \beta$, simulated in sim1c, proceeded quickly, as seen in Figure 5. The angle decreased by nearly $10^{\circ}$ in the first nanosecond of simulation, after which decrease was slow for the next 6 ns. However, in the following $1 \mathrm{~ns}$, the angle decreased further by $4^{\circ}$. The period of relative stability in this case is due to the lipids, in particular, the lipid tails at the gate. During gate opening, 


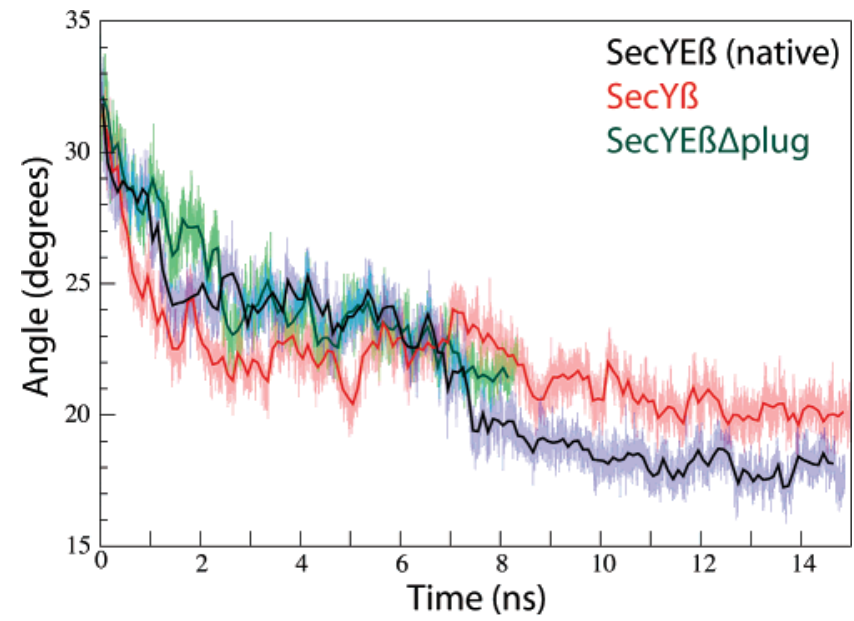

FIGURE 5: Relaxation after gate opening. The plot shows the angle of the gate opening (defined in Figure 2) as a function of time during simulated relaxation of each system, colored as indicated. The light, thick lines represent the original data, and the solid, thin lines represent averages taken over 100 ps windows.

lipid tails interact with the hydrophobic sides of the gate, although they do not invade the polar, hydrated channel; these tails have to first evacuate the gate region before full closure can occur. At the conclusion of the 15 ns simulation, the angle is $17.5^{\circ}$, only $2.5^{\circ}$ wider than the initial state. The rmsd of the protein compared to its original closed state, presented in the Supporting Information, also shows a marked decrease.

Relaxation of the other two structures simulated, $\operatorname{SecY} \beta$ $(\operatorname{sim} 2 b)$ and $\operatorname{SecYE} \beta \Delta$ plug $(\operatorname{sim} 3 b)$, progressed at a rate similar to that seen in sim1c (see Figure 5). Even after 15 $\mathrm{ns}$ for $\operatorname{Sec} \mathrm{Y} \beta$ and over $8 \mathrm{~ns}$ for $\operatorname{SecYE} \beta \Delta$ plug, the measured angles differ by no more than approximately $2^{\circ}$ from that of the native $\operatorname{Sec} Y E \beta$. This indicates that $\operatorname{SecE}$ (the previously suggested "clamp") does not significantly aid gate closure in the simulated system. Because the plug is most relevant when in contact with all gating helices, it is not surprising that the effect of removing it on the rate of gate closure is small.

Given the large resistance to gate opening as a result of the membrane as seen in the previous simulations, we asked if the membrane also plays a role in gate closing. We therefore simulated in sim1d the relaxation of the native $\operatorname{SecYE} \beta$ with the majority of lipid head groups restrained, thus determining if $\operatorname{Sec} \mathrm{YE} \beta$ would close on its own. As shown in Figure 6, the rate of closure in this simulation was found to be much slower in the first $6 \mathrm{~ns}$, at which point gate closure is reversed somewhat, because of interactions with the lipid tails at the interface. We note that lipids near the interface were not restrained and therefore were not prevented from exiting the gating region.

The flow of water across the channel during gate opening and closing was also analyzed. While one would expect the channel to be blocked well by a translocating or membraneinserting polypeptide, it still must be capable of closing after this process. During gate opening ( $\operatorname{sim} 1 \mathrm{a})$, the channel became hydrated, permitting the flow of 200 water molecules in the 6 ns simulation. However, during relaxation $(\operatorname{sim} 1 \mathrm{c})$, water permeation soon ended. While in the first $5 \mathrm{~ns}, 44$ water molecules cross the channel, in the next $10 \mathrm{~ns}$, only 7 manage to cross. Comparisons between simulations showed

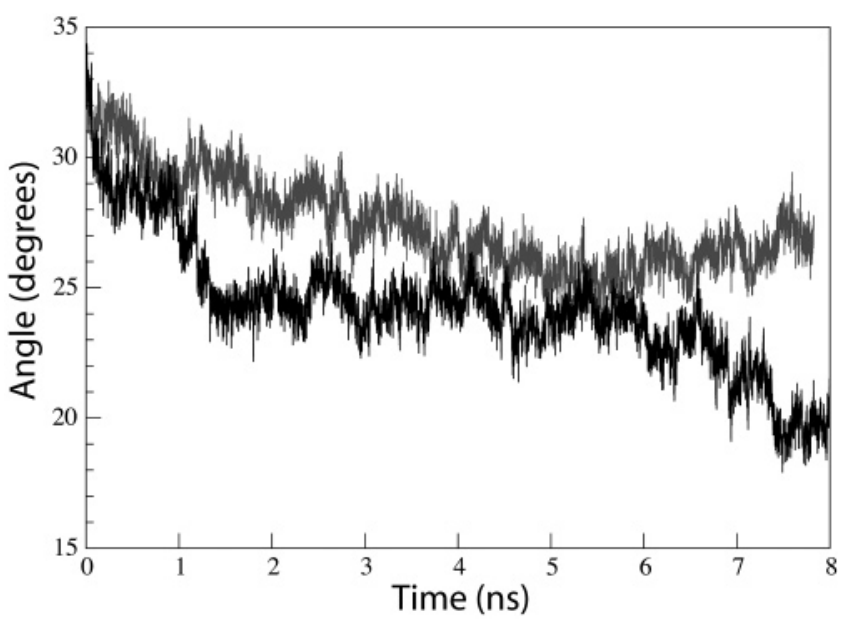

FIGURE 6: Relaxation of $\operatorname{SecYE} \beta$. The angle of gate opening is shown as a function of time for free relaxation (sim1c, black, see also Figure 5) as compared to simulation sim1d (gray), in which the lipids were restrained during relaxation.

the plug and, to a lesser degree, the pore ring, the hydrophobic constriction point at the center of the channel $(8,10)$, to be responsible for the rapid blockage of the channel.

Lipid Behavior near the Gate. It has been suggested recently that if the open lateral gate exposes the channel to the bilayer, it would permit an influx of lipids (23). While such influx was not seen in our simulations of gate opening and relaxation, we wondered if it was possible in general and, if not, what factors prevent it. Therefore, we performed simulations of the protein with the lateral gate held open to the bilayer, using both an all-atom and a CG representation of the protein-lipid-water system (see the Materials and Methods).

After the lateral gate of $\operatorname{SecYE} \beta$ (simulation sim1a) was forced apart, an opening 10-16 $\AA$ across remained enforced for simulation sim1e, as seen in Figure 7. In this simulation, the gate was held open for $20 \mathrm{~ns}$, while lipids were left free to diffuse. During the entire length of the simulation, no encroachment of an entire lipid into the channel is observed. As seen previously, lipid tails do continue to sample the gate region, and also, between 10 and $20 \mathrm{~ns}$ in the simulation, the head group of one lipid is observed to move toward the hydrated channel (see Figure 7). However, the tails of this lipid still remain deeply embedded in the bilayer and wrapped around the protein (a comparison of the behavior of these lipids to those surrounding a hole in a bilayer is presented in the Supporting Information). The plug largely blocks the lower (periplasmic) portion of the channel during the simulation, preventing any lipids in the lower bilayer leaflet from entering.

After $20 \mathrm{~ns}$ of holding the gate open, the protein was once again allowed to relax ( $\operatorname{sim} 1 \mathrm{~g})$. The angle of opening was tracked as a measure of closure and was compared to the relaxation performed in simulation sim $1 \mathrm{c}$. The two relaxation processes are shown in Figure 8. While the gate was slower to close in simulation sim $1 \mathrm{~g}$, relaxation still progressed in a qualitatively similar manner. After a brief (1 ns) period of fast closure, the gate remained at a fixed angle for approximately $6 \mathrm{~ns}$ because of the lipids still present at the membrane/gate interface. After these lipids left the interface, the angle steadily decreased again for the remainder of the $15 \mathrm{~ns}$ simulation, ending at $22^{\circ}$, i.e., at an angle $4.5^{\circ}$ larger 

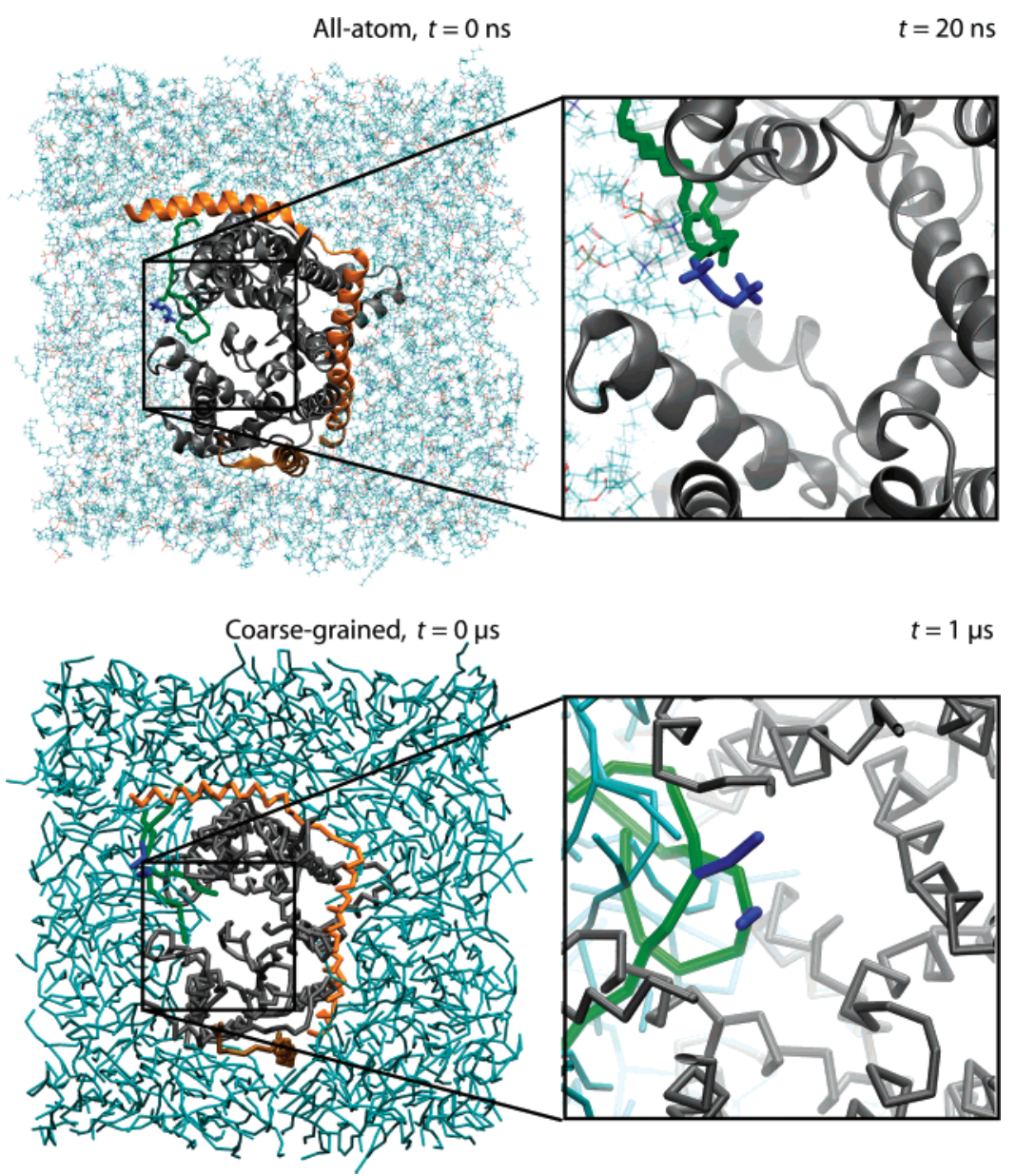

FIGURE 7: Behavior of lipids near the open lateral gate. In all frames, $\operatorname{SecYE} \beta$ is shown in cartoon representation, with SecY colored in gray, $\operatorname{SecE}$ colored in orange, and $\operatorname{Sec} \beta$ colored in brown. Individual lipids are shown in licorice, colored mostly light blue, with one or two specific lipids highlighted in blue (head group) and green (tails). In both top and bottom, the right frame represents a close-up view of the channel, with depth indicated by increasing transparency. (Top) Initial and final states for the all-atom system. The gate of SecY was held open to the bilayer for $20 \mathrm{~ns}$. (Bottom) Simulation repeated for a CG system. The gate was held open for $1 \mu \mathrm{s}$. In both cases, lipids made very little entry into the channel.

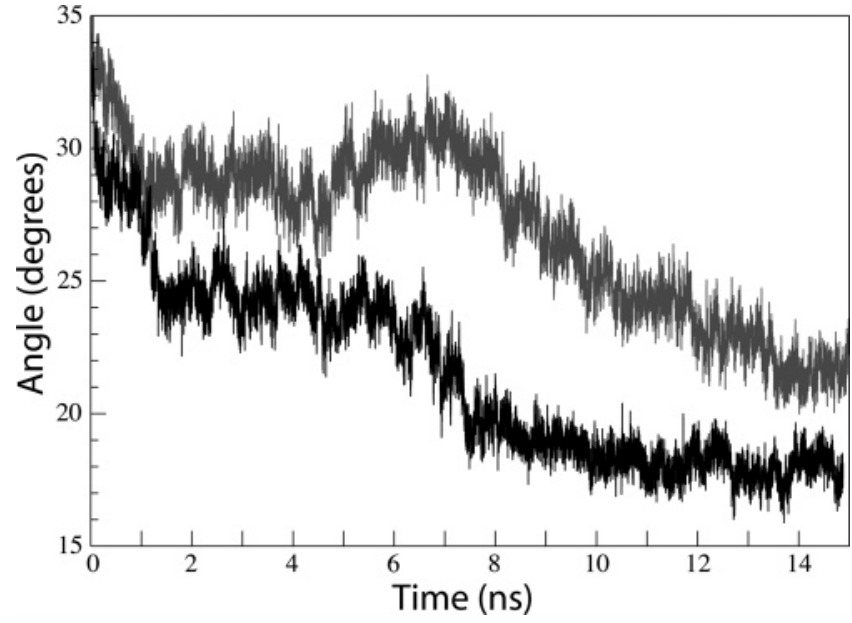

FIGURE 8: Relaxation of $\operatorname{SecYE} \beta$ after holding the gate open for $20 \mathrm{~ns}$. The angle of gate opening is shown as a function of time for relaxation immediately after gate opening (black, sim1c) and for relaxation of the protein after the gate was held open for $20 \mathrm{~ns}$ (gray, sim1g).

than that at the conclusion of sim1c. Thus, while lipids appear to block the gate slightly more after it was held open for 20 ns, they do not provide a significant barrier to closing.
Recognizing that the time scales of the all-atom simulations described previously may not be sufficient to describe the lipid behavior fully, a $1 \mu \mathrm{s}$ CG simulation was run (see the Materials and Methods). In this simulation, sim1CG, the setup used in simle was duplicated, artificially holding the gate in an open configuration. CG simulations have been used successfully to describe the properties of lipid-water systems and, more recently, of protein-lipid systems (35, 36); CG simulations of membrane protein insertion and interaction with lipids have also validated the usefulness of the method (55-57). On the basis of this success, it was believed that a CG simulation would accurately reproduce the hydrophilic/hydrophobic interactions that govern the lipid-protein dynamics near the lateral gate of SecY. In fact, the lipids in the CG simulation displayed a behavior similar to what was observed for the all-atom simulation, sim1e. After $1 \mu \mathrm{s}$, the head groups of two lipids were seen to partially enter the channel, although the tails remained trapped within the bilayer (see Figure 7). However, during the course of much of the microsecond simulation, only the head groups of a few lipids were observed to enter and exit the channel. Because the effective size of a CG bead is $\sim 4.7$ $\AA$ in diameter, for the lateral gate opening of between 10 and $16 \AA$, at least one lipid should be capable of crossing the gate at a time. 


\section{DISCUSSION}

Given the mechanical nature of $\operatorname{Sec} \mathrm{YE} \beta$, MD simulations can naturally present a clear view of the structural behavior during protein translocation and integration processes at an atomic level. Specifically, our simulations examined the structural changes that accompany opening and closing of the lateral gate of the protein translocon. We simulated SecYE $\beta$ (sim1a and sim1c) along with two modified systems in which we removed $\operatorname{SecE}(\operatorname{Sec} Y \beta$; $\operatorname{sim} 2 \mathrm{a}$ and $\operatorname{sim} 2 \mathrm{~b})$ or the plug (SecYE $\beta \Delta$ plug; sim $3 \mathrm{a}$ and $\operatorname{sim} 3 b)$. Because the lateral gate is the proposed location for insertion of membrane proteins, the motions induced in our simulations through forces applied to the sides of the gate should reasonably reflect those that the protein goes through during opening in vivo, although it still remains unknown what changes in the structure may be induced by the presence of a channel partner and the nascent chain.

The most unexpected result from the present simulations is that SecE, the smaller accessory protein, which had been presumed to form a clamp around the channel SecY, does not function as one. During simulated relaxation of the open state of the channel (simulations sim $1 c, \operatorname{sim} 2 b, \operatorname{sim} 3 b$, and $\operatorname{sim} 1 \mathrm{~g}$ ), the presence or absence of SecE had no effect on the rate of gate closure. In retrospect, this is not surprising: the linker region between the two helices in SecE is too soft to maintain a specific conformation. Much of the available experimental data appears to support this interpretation. While SecE is known to be necessary for translocation, it tolerates deletion of much of the N-terminal portion, including two additional TM helices present in Escherichia coli $(58,59)$. The required portion consists of a conserved cytoplasmic domain (approximately residues $71-89$ in $E$. coli) and the TM helix crossing the front of SecY (58-61). Figure $2 \mathrm{~A}$ shows the location of these residues on the structure (mapped from E. coli to the M. jannaschii structure), indicating that most of one side of the proposed clamp is not essential. Further experiments demonstrate that much of the N-terminal portion of SecE is important, not for function, but to prevent degradation of the complex by the protease FtsH $(62,63)$. The cytoplasmic domain, which accepts limited mutations, is probably required for proper association with $\mathrm{SecY}$ and/or other channel-binding partners $(59,60$, 64). The combination of the computational and experimental results indicates that the function of SecE may be to catalyze translocation (65), prevent degradation, or recruit channel partners, but it is unlikely to act as a clamp during membrane protein integration.

In the simulations, another structural element, the channelblocking plug of SecY, resisted lateral gate opening because of its hydrophobic contact with the gate helices. A destabilization of the plug in functional dimers of the channel had already been predicted by simulation, modeling, and experiment $(10,28,66)$. One early step in opening the channel, the insertion of the signal sequence, has been proposed to cause the lateral gate to open slightly, which then frees the plug (2). In our simulations, we observed that opening the gate to $\sim 24^{\circ}$ was sufficient to break the contact between the plug and the rest of the channel. It is known that the signal sequence contacts both TMs $2 \mathrm{~b}$ and 7 as well as lipids when it is inserted (67); at an intermediate gate opening, lipids were seen to sample the interior of the channel. It has also been hypothesized that insertion of the signal sequence is permitted because of "breathing modes" of the channel, in which the lateral gate opens and closes slightly (2). While in equilibrium, fluctuations of the gate on the order of only $2-4^{\circ}$ have been observed (whereas up to $10^{\circ}$ may be necessary), dimerization of the channel, the association of channel partners, or even the presence of the signal sequence may enhance such behavior.

Also investigated in the simulations was the behavior of lipids, especially near the gate. A lipid influx during membrane protein integration that could inactivate the channel was presented initially as one flaw of a back-toback dimer model of the channel $(23,29)$. We examined this proposal in simulations of gate opening and closing as well as ones in which the gate was held open to the bilayer. However, the behavior of lipids was not as dramatic as might have been expected. In all simulations, lipids did not invade the open channel, instead preferring to cling to the hydrophobic sides of the exposed gate helices. Favorable interactions between the lipids as well as the entropic penalty of one or two lipids crossing the gate likely both contribute to their behavior. While extension of our results (on the basis of tens of nanoseconds to $1 \mu \mathrm{s}$ of simulation) to the relevant time scales for protein insertion (on the order of seconds) is not straightforward, it is not clear that the interior of the channel would be exposed to the bilayer for seconds, because an inserting TM could occupy the gate for much of that time. On the basis of our analysis, it seems unlikely then that a lipid influx would actually occur on a scale large enough to inactivate the channel. The accessibility of lipids to a nascent polypeptide in the process of translocation is in agreement with experiments suggesting that interaction with the lipid bilayer helps to determine TM insertion $(12,68-71)$, although it is known that cooperative effects between TMs are sometimes necessary $(16-18)$. We suspect elements of $\mathrm{SecY}$, such as the hydrophobic pore ring, may also help to determine the placement of a TM in the bilayer.

All simulations performed reinforced the assumed mechanism for lateral gate opening, specifically, the suggestion of a clamshell-like structure of SecY, where the two halves of the protein are relatively independent during opening $(8$, 72); however, there appears to be little elastic behavior inherent in SecY or SecE that would maintain a closed conformation. Evidence of an initial step in the gating process, namely, the destabilization of the plug through gate opening, was observed; other steps such as a concomitant increase in the flexibility of the structure as a whole may follow (11). While it remains to be seen how dimerization or the binding of channel partners, such as the ribosome, would affect our observations, the conclusions arrived at are expected to be mostly independent of such partners.

\section{SUPPORTING INFORMATION AVAILABLE}

Data from additional simulations performed to test and/ or verify results presented here. This material is available free of charge via the Internet at http://pubs.acs.org.

\section{REFERENCES}

1. Veenendaal, A. K. J., van der Does, C., and Driessen, A. J. M. (2004) The protein-conducting channel SecYEG, Biochim. Biophys. Acta 1694, 81-95. 
2. Osborne, A. R., Rapoport, T. A., and van den Berg, B. (2005) Protein translocation by the Sec61/SecY channel, Annu. Rev. Cell Dev. Biol. 21, 529-550.

3. Pohlschröder, M., Hartmann, E., Hand, N. J., Dilks, K., and Haddad, A. (2005) Diversity and evolution of protein translocation, Annu. Rev. Microbiol. 59, 91-111.

4. Wickner, W., and Schekman, R. (2005) Protein translocation across biological membranes, Science 310, 1452-1456.

5. Luirink, J., von Heijne, G., Houben, E., and de Gier, J.-W. (2005) Biogenesis of inner membrane proteins in Escherichia coli, Annu. Rev. Microbiol. 59, 329-355.

6. Albers, S.-V., Szabó, Z., and Driessen, A. J. M. (2006) Protein secretion in the Archaea: Multiple paths towards a unique cell surface, Nat. Rev. Microbiol. 4, 537-547.

7. Matlack, K. E. S., Mothes, W., and Rapoport, T. A. (1998) Protein translocation: Tunnel vision, Cell 92, 381-390.

8. van den Berg, B., Clemons, W. M., Jr., Collinson, I., Modis, Y., Hartmann, E., Harrison, S. C., and Rapoport, T. A. (2004) X-ray structure of a protein-conducting channel, Nature 427, 36-44.

9. Cannon, K. S., Or, E., Clemons, W. M., Jr., Shibata, Y., and Rapoport, T. A. (2005) Disulfide bridge formation between SecY and a translocating polypeptide localizes the translocation pore to the center of SecY, J. Cell Biol. 169, 219-225.

10. Gumbart, J., and Schulten, K. (2006) Molecular dynamics studies of the archaeal translocon, Biophys. J. 90, 2356-2367.

11. Junne, T., Schwede, T., Goder, V., and Spiess, M. (2006) The plug domain of yeast Sec61p is important for efficient protein translocation, but is not essential for cell viability, Mol. Biol. Cell 17, 4063-4068.

12. Hessa, T., Kim, H., Bihlmaier, K., Lundin, C., Boekel, J., Andersson, H., Nilsson, I., White, S. H., and von Heijne, G. (2005) Recognition of transmembrane helices by the endoplasmic reticulum translocon, Nature 433, 377-381.

13. White, S. H., and von Heijne, G. (2005) Transmembrane helices before, during, and after insertion, Curr. Opin. Struct. Biol. 15 , 378-386.

14. Shental-Bechor, D., Fleishman, S. J., and Ben-Tal, N. (2006) Has the code for protein translocation been broken? Trends Biochem. Sci. 31, 192-196.

15. Mothes, W., Heinrich, S. U., Graf, R., Nilsson, I., von Heijne, G., Brunner, J., and Rapoport, T. A. (1997) Molecular mechanism of membrane protein integration into the endoplasmic reticulum, Cell 89, 523-533.

16. Sadlish, H., Pitonzo, D., Johnson, A. E., and Skach, W. R. (2005) Sequential triage of transmembrane segments by Sec61 $\alpha$ during biogenesis of a native multispanning membrane protein, Nat. Struct. Mol. Biol. 10, 870-878.

17. Ismail, N., Crawshaw, S. G., and High, S. (2006) Active and passive displacement of transmembrane domains both occur during opsin biogenesis at the Sec61 translocon, J. Cell Sci. 119, 28262836.

18. Zhang, L., Sato, Y., Hessa, T., von Heijne, G., Lee, J.-K., Kodama, I., Sakaguchi, M., and Uozumi, N. (2007) Contribution of hydrophobic and electrostatic interactions to the membrane integration of the shaker $\mathrm{K}^{+}$channel voltage sensor domain, Proc. Natl. Acad. Sci. U.S.A. 104, 8263-8268.

19. Oesterhelt, F., Oesterhelt, D., Pfeiffer, M., Engel, A., Gaub, H. E., and Müller, D. J. (2000) Unfolding pathways of individual bacteriorhodopsins, Science 288, 143-146.

20. Janovjak, H., Struckmeier, J., Hubain, M., Kedrov, A., Kessler, M., and Müller, D. J. (2004) Probing the energy landscape of the membrane protein bacteriorhodopsin, Structure 12, 871-879.

21. Breyton, C., Haase, W., Rapoport, T. A., Kühlbrandt, W., and Collinson, I. (2002) Three-dimensional structure of the bacteria protein-translocation complex SecYEG, Nature 418, 662-665.

22. Bessonneau, P., Besson, V., Collinson, I., and Duong, F. (2002) The SecYEG preprotein translocation channel is a conformationally dynamic and dimeric structure, EMBO J. 21, 995-1003.

23. Mitra, K., Schaffitzel, C., Shaikh, T., Tama, F., Jenni, S., Brooks, C. L., Ban, N., and Frank, J. (2005) Structure of the E. coli proteinconducting channel bound to a translating ribosome, Nature 438 , 318-324.

24. Hanein, D., Matlack, K. E. S., Jungnickel, B., Plath, K., Kalies, K.-U., Miller, K. R., Rapoport, T. A., and Akey, C. W. (1996) Oligomeric rings of the Sec61p complex induced by ligands required for protein translocation, Cell 87, 721-732.

25. Ménétret, J. F., Hegde, R. S., Heinrich, S. U., Chandramouli, P., Ludtke, S. J., Rapoport, T. A., and Akey, C. W. (2005)
Architecture of the ribosome-channel complex derived from native membranes, J. Mol. Biol. 348, 445-457.

26. Kaufmann, A., Manting, E. H., Veenendaal, A. K. J., Driessen, A. J. M., and van der Does, C. (1999) Cysteine-directed crosslinking demonstrates that helix 3 of SecE is close to helix 2 of SecY and helix 3 of a neighboring SecE, Biochemistry 38, 91159125.

27. Veenendaal, A. K., van der Does, C., and Driessen, A. J. (2001) Mapping the sites of interaction between SecY and SecE by cysteine scanning mutagenesis, J. Biol. Chem. 276, 32559-32566.

28. Bostina, M., Mohsin, B., Kühlbrandt, W., and Collinson, I. (2005) Atomic model of the $E$. coli membrane-bound protein translocation complex SecYEG, J. Mol. Biol. 352, 1035-1043.

29. Mitra, K., and Frank, J. (2006) A model for co-translational translocation: Ribosome-regulated nascent polypeptide translocation at the protein-conducting channel, FEBS Lett. 580, 33533360 .

30. Mitra, K., Frank, J., and Driessen, A. (2006) Co- and posttranslational translocation through the protein-conducting channel: Analogous mechanisms at work? Nat. Struct. Mol. Biol. 13, 957-964.

31. Humphrey, W., Dalke, A., and Schulten, K. (1996) VMD-Visual molecular dynamics, J. Mol. Graphics Modell. 14, 33-38.

32. Phillips, J. C., Braun, R., Wang, W., Gumbart, J., Tajkhorshid, E., Villa, E., Chipot, C., Skeel, R. D., Kale, L., and Schulten, K. (2005) Scalable molecular dynamics with NAMD, J. Comp. Chem. 26, 1781-1802.

33. MacKerell, A. D., Jr., Bashford, D., Bellott, M., Dunbrack, R. L., Jr., Evanseck, J., Field, M. J., Fischer, S., Gao, J., Guo, H., Ha, S., Joseph, D., Kuchnir, L., Kuczera, K., Lau, F. T. K., Mattos, C., Michnick, S., Ngo, T., Nguyen, D. T., Prodhom, B., Reiher, I. W. E., Roux, B., Schlenkrich, M., Smith, J., Stote, R., Straub, J., Watanabe, M., Wiorkiewicz-Kuczera, J., Yin, D., and Karplus, M. (1998) All-atom empirical potential for molecular modeling and dynamics studies of proteins, J. Phys. Chem. B 102, 35863616.

34. Marrink, S. J., de Vries, A. H., and Mark, A. E. (2004) Coarse grained model for semiquantitative lipid simulations, J. Phys. Chem. B 108, 750-760.

35. Shih, A. Y., Arkhipov, A., Freddolino, P. L., and Schulten, K. (2006) Coarse grained protein-lipid model with application to lipoprotein particles, J. Phys. Chem. B 110, 3674-3684.

36. Shih, A. Y., Freddolino, P. L., Arkhipov, A., and Schulten, K. (2007) Assembly of lipoprotein particles revealed by coarsegrained molecular dynamics simulations, J. Struct. Biol. 157, 579592.

37. Izrailev, S., Stepaniants, S., Balsera, M., Oono, Y., and Schulten, K. (1997) Molecular dynamics study of unbinding of the avidinbiotin complex, Biophys. J. 72, 1568-1581.

38. Isralewitz, B., Gao, M., and Schulten, K. (2001) Steered molecular dynamics and mechanical functions of proteins, Curr. Opin. Struct. Biol. 11, 224-230.

39. Puklin-Faucher, E., Gao, M., Schulten, K., and Vogel, V. (2006) How the headpiece hinge angle is opened: New insights into the dynamics of integrin activation, J. Cell Biol. 175, 349-360

40. Roberts, E., Eargle, J., Wright, D., and Luthey-Schulten, Z. (2006) MultiSeq: Unifying sequence and structure data for evolutionary analysis, BMC Bioinformatics 7, 382.

41. Izrailev, S., Stepaniants, S., Isralewitz, B., Kosztin, D., Lu, H., Molnar, F., Wriggers, W., and Schulten, K. (1998) Lecture notes in computational science and engineering, in Computational Molecular Dynamics: Challenges, Methods, Ideas (Deuflhard, P., Hermans, J., Leimkuhler, B., Mark, A. E., Reich, S., and Skeel, R. D., Eds.) Vol. 4, pp. 39-65, Springer-Verlag, Berlin, Germany.

42. Isralewitz, B., Baudry, J., Gullingsrud, J., Kosztin, D., and Schulten, K. (2001) Steered molecular dynamics investigations of protein function, J. Mol. Graphics Modell. 19, 13-25.

43. Gao, M., Sotomayor, M., Villa, E., Lee, E., and Schulten, K. (2006) Molecular mechanisms of cellular mechanics, Phys. Chem. Chem. Phys. 8, 3692-3706.

44. Marszalek, P. E., Lu, H., Li, H., Carrion-Vazquez, M., Oberhauser, A. F., Schulten, K., and Fernandez, J. M. (1999) Mechanical unfolding intermediates in titin modules, Nature 402, 100-103.

45. Gao, M., Craig, D., Lequin, O., Campbell, I. D., Vogel, V., and Schulten, K. (2003) Structure and functional significance of mechanically unfolded fibronectin type III1 intermediates, Proc. Natl. Acad. Sci. U.S.A. 100, 14784-14789. 
46. Sotomayor, M., Corey, D. P., and Schulten, K. (2005) In search of the hair-cell gating spring: Elastic properties of ankyrin and cadherin repeats, Structure 13, 669-682.

47. Lee, G., Abdi, K., Jiang, Y., Michaely, P., Bennett, V., and Marszalek, P. E. (2006) Nanospring behaviour of ankyrin repeats, Nature 440, 246-249.

48. Li, L., Wetzel, S., Pluckthun, A., and Fernandez, J. M. (2006) Stepwise unfolding of ankyrin repeats in a single protein revealed by atomic force microscopy, Biophys. J. 90, L30-L32.

49. Sotomayor, M., and Schulten, K. (2007) Single-molecule experiments in vitro and in silico, Science 316, 1144-1148.

50. Tian, P., and Andricioaei, I. (2006) Size, motion, and function of the SecY translocon revealed by molecular dynamics simulations with virtual probes, Biophys. J. 90, 2718-2730.

51. Haider, S., Hall, B. A., and Sansom, M. S. P. (2006) Simulations of a protein translocation pore: SecY, Biochemistry 45, 1301813024.

52. Wriggers, W., and Schulten, K. (1997) Protein domain movements: Detection of rigid domains and visualization of hinges in comparisons of atomic coordinates, Proteins: Struct., Funct., Genet. 29, 1-14.

53. Hayward, S., Kitao, A., and Berendsen, H. J. C. (1997) Modelfree methods of analyzing domain motions in proteins from simulation: A comparison of normal mode analysis and molecular dynamics simulation of lysozyme, Proteins: Struct., Funct., Genet. 27, 425-437.

54. Vallone, B., Miele, A. E., Vecchini, P., Chiancone, E., and Brunori, M. (1998) Free energy of burying hydrophobic residues in the interface between protein subunits, Proc. Natl. Acad. Sci. U.S.A. 95, 6103-6107.

55. Bond, P. J., and Sansom, M. S. P. (2006) Insertion and assembly of membrane proteins via simulation, J. Am. Chem. Soc. 128, 2697-2704.

56. Bond, P. J., Holyoake, J., Ivetac, A., Khalid, S., and Sansom, M. (2007) Coarse-grained molecular dynamics simulations of membrane proteins and peptides, J. Struct. Biol. 157, 593-605.

57. Bond, P. J., and Sansom, M. (2007) Bilayer deformation by the $\mathrm{Kv}$ channel voltage sensor domain revealed by self-assembly simulations, Proc. Natl. Acad. Sci. U.S.A. 104, 2631-2636.

58. Schatz, P. J., Bieker, K. L., Ottemann, K. M., Silhavy, T. J., and Beckwith, J. (1991) One of three transmembrane stretches is sufficient for the functioning of the SecE protein, a membrane component of the E. coli secretion machinery, EMBO J. 10, 17491757.

59. Nishiyama, K., Mizushima, S., and Tokuda, H. (1992) The carboxyl-terminal region of SecE interacts with SecY and is functional in the reconstitution of protein translocation activity in Escherichia coli, J. Biol. Chem. 267, 7170-7176.
60. Murphy, C. K., and Beckwith, J. (1994) Residues essential for the function of SecE, a membrane component of the Escherichia coli secretion apparatus, are located in a conserved cytoplasmic region, Proc. Natl. Acad. Sci. U.S.A. 91, 2557-2561.

61. Matsuo, E., Mori, H., and Ito, K. (2003) Interfering mutations provide in vivo evidence that Escherichia coli $\mathrm{SecE}$ functions in multimeric states, Mol. Gen. Genomics 268, 808-815.

62. Akiyama, Y., Kihara, A., Tokuda, H., and Ito, K. (1996) FtsH (HflB) is an ATP-dependent protease selectively acting on SecY and some other membrane proteins, J. Biol. Chem. 271, 3119631201 .

63. Nishiyama, K., Suzuki, H., and Tokuda, H. (2000) Role of the non-essential region encompassing the N-terminal two transmembrane stretches of Escherichia coli SecE, Biosci., Biotechnol., Biochem. 64, 2121-2127.

64. Pohlschröder, M., Murphy, C., and Beckwith, J. (1996) In vivo analyses of interactions between SecE and SecY, core components of the Escherichia coli protein translocation machinery, J. Biol. Chem. 271, 19908-19914.

65. Swaving, J., van Wely, K. H. M., and Driessen, A. J. M. (1999) Preprotein translocation by a hybrid translocase composed of Escherichia coli and Bacillus subtilis subunits, J. Bacteriol. 181 $7021-7027$.

66. Tam, P. C. K., Maillard, A. P., Chan, K. K. Y., and Duong, F. (2005) Investigating the SecY plug movement at the SecYEG translocation channel, EMBO J. 24, 3380-3388.

67. Plath, K., Mothes, W., Wilkinson, B. M., Stirling, C. J., and Rapoport, T. A. (1998) Signal sequence recognition in posttranslational protein transport across the yeast ER membrane, Cell 94, 795-807.

68. Martoglio, B., Hofmann, M. W., Brunner, J., and Dobberstein, B. (1995) The protein-conducting channel in the membrane of the endoplasmic reticulum is open laterally toward the lipid bilayer, Cell 81, 207-214.

69. Heinrich, S. U., Mothes, W., Brunner, J., and Rapoport, T. A (2000) The Sec61p complex mediates the integration of a membrane protein by allowing lipid partitioning of the transmembrane domain, Cell 102, 233-244.

70. Higy, M., Gander, S., and Spiess, M. (2005) Probing the environment of signal-anchor sequences during topogenesis in the endoplasmic reticulum, Biochemistry 44, 2039-2047.

71. White, S. H. (2007) Membrane protein integration: The biologyphysics nexus, J. Gen. Physiol. 129, 363-369.

72. Clemons, W. M., Jr., Ménétret, J. F., Akey, C. W., and Rapoport, T. A. (2004) Structural insight into the protein translocation channel, Curr. Opin. Struct. Biol. 14, 390-396. 